\title{
Cognitive dissonance in children: Justification of effort or contrast?
}

\author{
Jérôme Alessandri And Jean-Claude Darcheville \\ University of Lille III, Lille, France \\ AND \\ ThOMAS R. ZENTALL \\ University of Kentucky, Lexington, Kentucky
}

\begin{abstract}
Justification of effort is a form of cognitive dissonance in which the subjective value of an outcome is directly related to the effort that went into obtaining it. However, it is likely that in social contexts (such as the requirements for joining a group) an inference can be made (perhaps incorrectly) that an outcome that requires greater effort to obtain in fact has greater value. Here we present evidence that a cognitive dissonance effect can be found in children under conditions that offer better control for the social value of the outcome. This effect is quite similar to contrast effects that recently have been studied in animals. We suggest that contrast between the effort required to obtain the outcome and the outcome itself provides a more parsimonious account of this phenomenon and perhaps other related cognitive dissonance phenomena as well. Research will be needed to identify cognitive dissonance processes that are different from contrast effects of this kind.
\end{abstract}

Cognitive dissonance can be defined as the conflict that arises when there is an inconsistency between one's beliefs and one's behavior, or between two cognitions. According to cognitive dissonance theory (Festinger, 1957), one should be motivated to reduce that conflict, generally by altering one's beliefs.

Justification of effort is a form of cognitive dissonance in which one gives greater value to outcomes that require greater effort to obtain, to justify the greater effort (Aronson \& Mills, 1959). For example, a student receiving an A grade in a difficult course, such as organic chemistry, is likely to value that grade more than the same outcome in a less demanding course, such as an introduction to golf. According to cognitive dissonance theory, greater value is attributed to the outcome following greater effort, to justify the added effort needed to obtain it.

The problem with the justification-of-effort effect when applied to social contexts is that the assumption of "same outcome" may not be correct. Although A grades in chemistry and in golf may contribute equally to the student's grade-point average, the chemistry grade is likely to have greater value on an application to medical school.

Similarly, in the classic experiment by Aronson and Mills (1959), participants were told that they could take part in a discussion group if they passed an initiation consisting of reading a passage out loud. Aronson and Mills found that participants who were asked to read an embarrassing (sexually explicit) passage (a severe initiation) valued joining the group more than did participants for whom the passage was not so embarrassing (an easier initiation). However, participants may have applied a notinappropriate rule of thumb that presumes that groups that are difficult (or embarrassing) to join are generally more attractive socially (i.e., are generally valued more) than are groups that are easy to join.

Other interpretations of the results of this experiment have been proposed. For example, Gerard and Mathewson (1966) proposed that aversiveness in the Aronson and Mills (1959) experiment may have been confounded with (sexual) arousal in the difficult initiation condition, but they found that similar effects could be obtained with shock as the severe initiation.

Alternatively, Schopler and Bateson (1962) argued that because participants had already accepted to engage in a severe initiation, they were more likely to conform to the implied expectation that they should value the discussion group. Schopler and Bateson noted that dissonance theory would have predicted that participants in the severe initiation condition who felt most embarrassed by the initiation should have rated the discussion group most favorably; instead, they found the opposite. Thus, they suggested that reporting favorably on the group may have been perceived as a demand characteristic of the task resulting from the difficult initiation.

A third interpretation was proposed by Lodewijkx and Syroit (1997). On the basis of affiliation theory, they suggested that people under threat (presumed to be so in the severe initiation) have a strong need for affiliation; thus,

T. R.Zentall, zentall@uky.edu 
their strong need to join the group led to a more favorable evaluation of the group (see also Lodewijkx \& Syroit, 2001, and Lodewijkx, van Zomeren, \& Syroit, 2005). In support of this hypothesis, Gump and Kulik (1997) found that participants exposed to threat showed an increase in the desire to affiliate.

In all of these studies, complex social interactions make it difficult to isolate potentially simpler mechanisms that might contribute to, or even account for, the justification-ofeffort effect. One way to provide a simpler test of the mechanism responsible for this effect is to remove some of the more complex social components of the testing procedure. For example, one can vary the effort required by participants to obtain a stimulus associated with an asocial reinforcer.

Clement, Feltus, Kaiser, and Zentall (2000) described such a procedure in an experiment with pigeons. Pigeons were trained to peck at a circle. On half of the trials, one peck was sufficient to remove the circle and present the pigeons with a simultaneous color discrimination (e.g., red + , yellow - ). On the remaining trials, the pigeons had to make 20 pecks to remove the circle and present a different simultaneous discrimination (e.g., green + , blue-). On test trials, when the pigeons were given a choice between the two $\mathrm{S}+$ stimuli (i.e., red and green) they showed a significant preference for the $\mathrm{S}+$ that in training had followed the greater effort (20 pecks). Similarly, DiGian, Friedrich, and Zentall (2004) found that when pigeons were trained to choose one $\mathrm{S}+$ stimulus following a 6-sec delay and a different $\mathrm{S}+$ stimulus following no delay, they preferred the $\mathrm{S}+$ that in training was preceded by the delay. And Friedrich, Clement, and Zentall (2005) reported that when pigeons were trained to choose one $\mathrm{S}+$ stimulus following food reinforcement and a different $\mathrm{S}+$ stimulus following the absence of food reinforcement, they preferred the $\mathrm{S}+$ that in training was preceded by the absence of reinforcement. Thus, in general, pigeons appear to prefer a conditioned reinforcer $(\mathrm{S}+)$ that follows a less preferred event to one that follows a more preferred event.

Klein, Bhatt, and Zentall (2005) conducted an experiment with adult humans similar to that undertaken by Clement et al. (2000), in which a single mouse click was required on half of the trials to produce one pair of shapes and 20-30 mouse clicks were required to produce a different pair of shapes on the remaining trials. In each case, participants had to learn by trial and error which of the two shapes in each pair was the correct one. Following acquisition, when the participants were given a choice between the two correct shapes, one from each discrimination, they showed a significant preference for the correct shape that had followed the greater response requirement in training. These results, together with those from experiments with pigeons, suggest that the justification-of-effort effect can be found in the absence of a social context (i.e., a context in which the outcome following the more difficult initiation may be inferred to be more valuable).

Clement et al. (2000) suggested that such preferences could be more parsimoniously explained in terms of a contrast effect. They suggested that contrast between the prior effort (or delay or absence of reinforcement) and the discriminative stimulus (signaling reinforcement) that followed could account for this effect. Furthermore, they suggested that experience with any less preferred event that occurred prior to presentation of a discriminative stimulus associated with reinforcement would result in a preference for that discriminative stimulus over one that followed a more preferred event.

Although the results found with adults suggest that the justification-of-effort effect may be found in an asocial context, the extensive history that adults have had in making judgments of the value of reinforcers in terms of the effort required to obtain them (i.e., requiring greater effort often implies that the reinforcer is of better quality) may make it difficult to isolate the mechanism responsible for this effect, even when studied in contexts that are not social. Finding a similar effect in young children-who have had less experience with the expectation that reinforcers that follow greater effort are actually worth more than those that follow less effort-would better indicate that the outcomes were actually similar.

Furthermore, if the added value of reinforcement depends on the presence of a prior aversive event, it should be possible to observe a similar effect with a different sort of prior aversive event, such as delay. Thus, a second purpose of the present experiment was to manipulate the delay (known to be a relatively aversive event; Fantino, 1969) between an initial common response and the reinforcer-associated stimulus that follows.

\section{METHOD}

\section{Participants}

Forty-two children (24 males and 18 females), age range 7-8 years, participated in the experiment. All were students from two typical classrooms enrolled in two classes in a public elementary school in Hem (a suburb of Lille), France.

\section{Apparatus}

The study was conducted in a quiet room in the school, close to the children's classroom. Each participant was seated on a chair at a table on which was a monitor connected to a computer. All participants were trained and tested with a program created with Labview 7.1 (National Instruments Corporation, Austin, Texas). During experimental sessions, the monitor displayed an initial stimulus at the top center of the screen and two discriminative stimuli near the bottom, one on each side, each measuring approximately $5 \times 5 \mathrm{~cm}$. Clicking the mouse within these areas advanced the trial sequence and resulted in programmed consequences. The initial stimuli used were red or blue rectangles. The discriminative stimuli that followed were familiar black shapes (plus, square, circle, and triangle) presented on a white background. The children were tested individually. They had no interaction with other participants before or during the experimental session, and we asked them not to talk to the other children about the experiment.

\section{Procedure}

Reinforcement preference assessment. Because of potential individual differences in the value of the rewards used, a preference assessment was conducted to determine for each participant the most preferred among three potential reinforcers, and one potential punisher (a time-out period). We selected reinforcers that we thought the children would like. The reinforcers were the 5 -sec presentation of (1) short segments of nine possible songs; (2) short segments of a French cartoon (Razmoket or Titeuf); (3) a voice that 
said "good," or (4) the blank screen alone (time-out). The child was then asked which event was the most preferred (to be used following choice of the positive, $\mathrm{S}+$, discriminative stimulus in training) and which was the least preferred (to be used following choice of the negative, $\mathrm{S}-$, discriminative stimulus in training). The participants were exposed to each reinforcer (two exposures to each reinforcer) signaled by an icon presented in the center of the screen. Thirty-eight of the children rated one of the cartoons as most preferred; the remaining children preferred the songs. All children rated the time-out as the least preferred.

Pretraining. After the preference assessment test, the children were given an example of the procedure in which a white rectangle and a black rectangle (not presented in training) were used as choice stimuli. Each child was told to click on each of the sample stimuli to see what outcomes would result. There were two pretraining trials presented; one for which a click on one shape produced the reinforcer, and another for which a click provided the time-out.

Training. Participants were told to click on the initial red or blue rectangle several times (seven clicks were required on each trial to make it disappear and to display the two shapes). They then had to discover which shape would produce the reinforcer (called the good outcome) by clicking on one of them. Each of the two discriminations involved two of the shapes (plus, square, circle, and triangle), chosen randomly for each child, with one shape chosen arbitrarily as the correct one $(\mathrm{S}+)$. Choice of the $\mathrm{S}+$ was followed by the most preferred event; choice of the other shape, the $\mathrm{S}-$, was followed by the least preferred event. On half the trials, a response to the colored rectangle was followed immediately with the presentation of one pair of shapes. On the remaining trials, a response to the other colored rectangle was followed by a delay of $8 \mathrm{sec}$ and then the presentation of the other pair of shapes (see Figure 1 for a schematic of the training procedure). Participants were told that if the shapes did not appear immediately, they should wait and not click any more until the shapes appeared. The experimenter then asked the participants whether they understood what they had to do. If not, the relevant portions of the instructions were repeated.

The two types of trial (with and without delay) were presented randomly, with the constraint that each type of trial occurred equally often in a block of four trials. The position of the shapes in each discrimination (left or right) was randomized, with the constraint that each stimulus occurred equally often on each side. There was an intertrial interval (ITI) of $1 \mathrm{sec}$, selected to be relatively short because the children became restless if the ITI was longer. Training continued until there were 16 consecutive responses to the correct shapes, typically 8 correct responses for each pair of discriminative stimuli.

Testing. Following training, the children were told that they should now choose which shape they most preferred, but that neither reward nor blackout would be delivered. Testing consisted of 12 test trials, 6 test trials with the simultaneous presentation of the two $\mathrm{S}+$ stimuli from training, and 6 test trials with the simultaneous presentation of the two $\mathrm{S}-$ stimuli from training (see Figure 2). The position of the two stimuli was randomized, with the constraint that each stimulus appeared equally often on each side. To assess the possibility that test stimulus preference might be conditional on the delay presented on test trials (rather than attributable to delay experienced in training), there were 3 possible initial events that preceded the test pair (each presented twice with the $\mathrm{S}+$ stimuli and the $\mathrm{S}-$ stimuli). The initial event was either the blue or red rectangle followed by the associated delay, or no initial event (choice between the $\mathrm{S}+$ or $\mathrm{S}-$ shapes occurred immediately following the ITI). The sequence of trials during testing was counterbalanced over participants.

\section{RESULTS}

\section{Testing}

Choice of the $\mathbf{S}+$. On test trials involving choice of the two $\mathrm{S}+$ stimuli, the children showed a preference for the $\mathrm{S}+$ that in training followed the delay $(62.3 \%, S E M=$
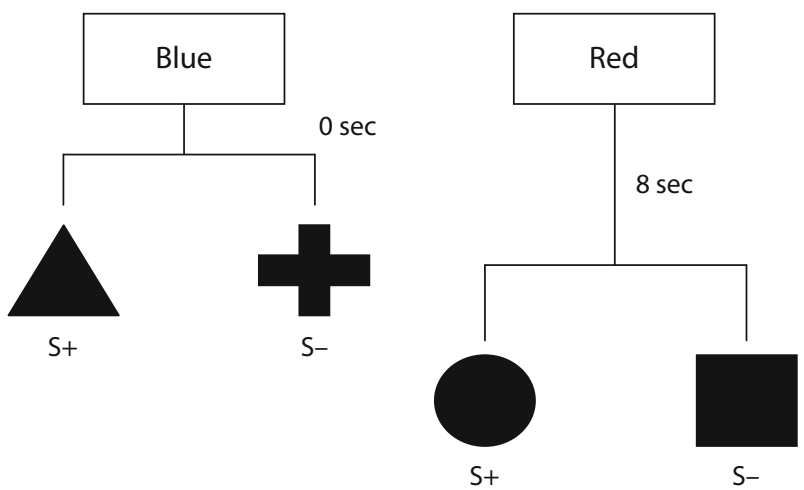

Figure 1. Sequence of events experienced by children in the nodelay component (left panel) and in the delay component (right panel) of the task during training. The colors that signaled the initial event and the discriminative stimuli associated with the delay and no-delay events were counterbalanced over children.

4.46). A single-sample $t$ test was conducted on the overall choice of the $\mathrm{S}+$ stimuli on test trials. The analysis indicated that choice of the delayed $\mathrm{S}+$ was significantly greater than chance $[t(41)=2.76, p<.01$, Cohen's $d=$ $.86]$, indicating that the $\mathrm{S}+{ }_{\text {delay }}$ stimulus was preferred over the $\mathrm{S}+_{\text {no delay }}$ stimulus.

On test trials, the children chose the $\mathrm{S}+{ }_{\text {delay }}$ when the initial event on test trials was the delay stimulus, $66.7 \%$ $(S E M=.6 .0)$; when the initial event on test trials was the no-delay stimulus, $54.8 \%(S E M=6.0)$; and when the initial event on test trials was no event, $65.5 \%(S E M=6.0)$. A one-way ANOVA performed on the test data, with the three levels of initial event on test trials (delay, no delay, and no event) as the factor, yielded a nonsignificant effect of the initial event on test trials $[F(2,82)=1.71, p=$ .19]. Thus, there was no significant evidence that the children had acquired a conditional discrimination in training. That is, in training they did not learn to choose the $\mathrm{S}+_{\text {delay }}$
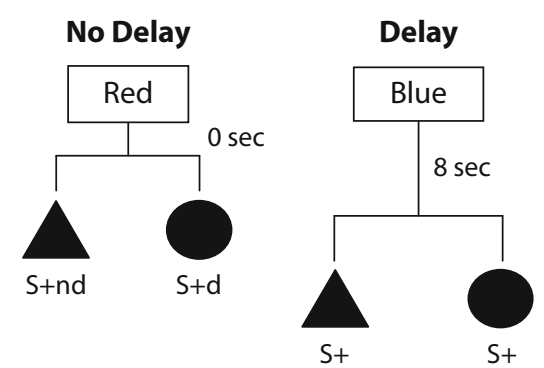

No Event

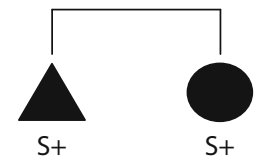

Figure 2. Sequence of events experienced by children in each initial stimulus condition for $\mathrm{S}+$ presentations during testing. On one third of the test trials, the children experienced an initial color followed by the 8-sec delay and either the two $S+$ stimuli from training (left panel) or the two $S-$ stimuli from training (not shown). On one third of the test trials, the children experienced an initial color followed by no delay, and either the two $S+$ from training (right panel) or the two $\mathrm{S}-$ stimuli from training (not shown). And on the remaining third of the test trials, the children experienced no initial stimulus and no delay, followed by either the two $S+$ from training (middle panel) or the two $S-$ stimuli from training (not shown). 
if there was a delay on the current trial or to choose the $\mathrm{S}+{ }_{\text {no delay }}$ in the event of no delay.

Choice of the $\mathbf{S}-$. On test trials involving choice of the two $\mathrm{S}-$ stimuli, the children showed a small preference for the $\mathrm{S}-$ delay $(55.5 \%, S E M=4.0)$. A single-sample $t$ test conducted on the overall choice of the $\mathrm{S}-{ }_{\text {delay }}$ on test trials indicated that choice of the delayed $\mathrm{S}-$ was not significantly different from chance $[t(41)=1.50, p=.14$, Cohen's $d=.47$ ].

On test trials, the children chose the $\mathrm{S}-{ }_{\text {delay }}$ when the initial event on test trials was the delay stimulus, $61.9 \%$ $(S E M=12.0)$; when the initial event on test trials was the no-delay stimulus, $50.0 \%(S E M=13.0)$; and when the initial event on test trials was no event, 61.9\% (SEM = 13.0). A one-way ANOVA performed on the test data, with the three levels of initial event on test trials (delay, no delay, and no event) as the factor, yielded a nonsignificant effect of the initial event on test trials $[F(2,82)=$ $2.40, p=.10]$. Thus, the initial event from training did not significantly affect choice of the two $\mathrm{S}-$ stimuli when it was presented on test trials.

\section{DISCUSSION}

The results of the present experiment show that children preferred the $\mathrm{S}+$ that followed the 8-sec delay in training over the $\mathrm{S}+$ that followed no delay, and that this effect was independent of whether the test-trial choice was preceded by a delay or not. This result confirms the finding that a cognitive dissonance or justification-of-effort effect can be obtained in the context of arbitrary stimuli that are presented in a relatively asocial context. A social context such as that used by Aronson and Mills (1959) may elicit learned strategies that can evoke unintended differences in the value of reinforcement. Humans often experience better outcomes when they require more effort to obtain them. Although such expectations may occur in young children as well, they are less likely to be present in an asocial context.

The fact that a very similar effect can be found in pigeons (see Zentall \& Singer, 2007, for a review) under virtually the same conditions as those used with both adults and children makes the assumption about an asocial context more credible. As noted, pigeons not only prefer stimuli that follow longer delays (DiGian et al., 2004), they also prefer stimuli that follow greater effort (Clement et al., 2000) and the absence of food (in the context of food on other trials; Friedrich et al., 2005). When this effect has been found in pigeons, it has been explained in terms of the contrast between the negative value of the aversive delay, effort, or absence of food and the positive value of the reinforcer, compared with the value of the absence of a delay, less effort, or presence of food and the positive value of the reinforcer.

In the present experiment, children were given a simultaneous discrimination following the delay or absence of a delay to ensure that they attended to the shapes and did not respond simply to any shape that appeared (see
Clement et al., 2000). On test trials, our main interest was which of the two $\mathrm{S}+$ stimuli the children preferred, but we also asked them to choose between the two $\mathrm{S}-$ stimuli. In research with pigeons, we have sometimes found a preference for the $\mathrm{S}-$ stimulus similar to the preference for the $\mathrm{S}+$ stimulus that followed the less-preferred event in training (Clement et al., 2000), but in several other experiments a preference for the $\mathrm{S}-$ stimulus has not been found (DiGian et al., 2004; Friedrich et al., 2005). When we have sometimes found such an $\mathrm{S}$ - preference, we have explained it in terms of value that may transfer from an $\mathrm{S}+$ to an $\mathrm{S}-$ in a simultaneous discrimination (Zentall \& Clement, 2001). However, we have also suggested that the reason the $\mathrm{S}-$ effect is often not found is that reliable value transfer effects occur primarily when the $\mathrm{S}+$ stimuli themselves have a greater difference in absolute value (e.g., differential probability of reinforcement) than they do in the present design.

Contrast effects have been studied in animals for over 50 years. They can be seen when there is an upward or downward shift in the magnitude of reinforcement relative to controls trained at the final level from the start (incentive contrast; Crespi, 1942), or when responding in the presence of one stimulus is extinguished and responding increases in the presence of a second stimulus without a change in its schedule of reinforcement associated with the second (behavioral contrast; Reynolds, 1961). Presumably, contrast effects are based on a simple comparative process, the degree of change in the value of an event, and do not require a cognitive mechanism such as the need to justify one's behavior or the dissonance between one's beliefs and one's behavior.

The fact that the present effect can be found in pigeons (under a variety of conditions), as well as in human children and adults, suggests that it has considerable generality. It also offers support for the hypothesis that findings that have been interpreted in terms of the justification of effort and attributed to complex processes such as the reduction in psychological discomfort produced by attitude change (Elliot \& Devine, 1994), an increased need to conform to the perceived expectation of the experimenter, or an increased need to affiliate may actually be based on a more parsimonious contrast mechanism. It is also possible that contrast is the basic underlying process responsible for this effect, but that various social variables, including cognitive dissonance, need to conform, and need for affiliation, reinforce the response tendencies established by contrast. If so, it would be of interest to determine the contribution of each of these mechanisms to this broad class of cognitive dissonance phenomena often thought of as paradoxical.

\section{AUTHOR NOTE}

This research was facilitated by a fellowship from the Fulbright Scholar Program and the Nord-Pas de Calais Regional Council, as well as by a visiting professorship at the University of Lille III for T.R.Z. Preparation of the article was facilitated by National Institute of Mental Health Grant MH 63726 to T.R.Z. Correspondence concerning this article should be addressed to T. R. Zentall, Department of Psychology, University of Kentucky, Lexington, KY 40506 (e-mail: zentall@uky.edu). 


\section{REFERENCES}

Aronson, E., \& Mills, J. (1959). The effect of severity of initiation on liking for a group. Journal of Abnormal \& Social Psychology, 59, $177-181$.

Clement, T. S., Feltus, J. R., Kaiser, D. H., \& Zentall, T. R. (2000). "Work ethic" in pigeons: Reward value is directly related to the effort or time required to obtain the reward. Psychonomic Bulletin \& Review, 7, 100-106

CRESPI, L. P. (1942). Quantitative variation in incentive and performance in the white rat. American Journal of Psychology, 55, 467-517.

DiGian, K. A., Friedrich, A. M., \& Zentall, T. R. (2004). Discriminative stimuli that follow a delay have added value for pigeons. Psychonomic Bulletin \& Review, 11, 889-895.

Elliot, A. J., \& Devine, P. G. (1994). On the motivational nature of cognitive dissonance: Dissonance as psychological discomfort. Journal of Personality \& Social Psychology, 67, 382-394.

FAntino, E. (1969). Choice and rate of reinforcement. Journal of the Experimental Analysis of Behavior, 12, 723-730.

Festinger, L. (1957). A theory of cognitive dissonance. Oxford: Row, Peterson.

Friedrich, A. M., Clement, T. S., \& Zentall, T. R. (2005). Discriminative stimuli that follow the absence of reinforcement are preferred by pigeons over those that follow reinforcement. Learning \& Behavior, 33, 337-342.

Gerard, H. B., \& Mathewson, G. C. (1966). The effects of severity of initiation on liking for a group: A replication. Journal of Experimental Social Psychology, 2, 278-287.

Gump, B. B., \& KulIK, J. A. (1997). Stress, affiliation, and emotional contagion. Journal of Personality \& Social Psychology, 72, 305-319.
Klein, E. D., Bhatt, R. S., \& Zentall, T. R. (2005). Contrast and the justification of effort. Psychonomic Bulletin \& Review, 12, 335-339.

LodewiJkx, H. F. M., \& Syroit, J. E. M. M. (1997). Severity of initiation revisited: Does severity of initiation increase attractiveness in real groups? European Journal of Social Psychology, 27, 275-300.

LodewiJkx, H. F. M., \& Syroit, J. E. M. M. (2001). Affiliation during naturalistic severe and mild initiations: Some further evidence against the severity-attraction hypothesis. Current Research in Social Psychology, 6, 90-107.

LodewiJkx, H. F. M., van Zomeren, M., \& Syroit, J. E. M. M. (2005). The anticipation of a severe initiation: Gender differences in effects on affiliation tendency and group attraction. Small Group Research, 36, 237-262.

REYNOLDS, G. S. (1961). Behavioral contrast. Journal of the Experimental Analysis of Behavior, 4, 57-71.

SCHOPLer, J., \& BATESON, N. (1962). A dependence interpretation of the effects of a severe initiation. Journal of Personality, 30, 633-649.

Zentall, T. R., \& Clement, T. S. (2001). Simultaneous discrimination learning: Stimulus interactions. Animal Learning \& Behavior, 29, 311-325.

Zentall, T. R., \& Singer, R. A. (2007). Within-trial contrast: Pigeons prefer conditioned reinforcers that follow a relatively more rather than less aversive event. Journal of the Experimental Analysis of Behavior, 88, 131-149.

(Manuscript received July 23, 2007; revision accepted for publication November 12, 2007.) 